\title{
Adipose tissue inflammation in human obesity and response to chronic marine omega-3 fatty acid supplementation: the BIOCLAIMS study at the University of Southampton
}

\author{
H. Fisk ${ }^{1}$, R. Ayres ${ }^{1}$, C. Childs ${ }^{1}$, O. Kuda ${ }^{2}$, J. Kopecky ${ }^{3}$, E. Antoun ${ }^{1}$, K. Lillycrop ${ }^{1}$ and \\ P. Calder ${ }^{1}$ \\ ${ }^{1}$ Human Development and Health Academic Unit, Faculty of Medicine, University of Southampton, Southampton, UK, \\ ${ }^{2}$ Department of Adipose Tissue Biology, Institute of Physiology of the Czech Academy of Sciences, Prague, Czech \\ Republic and \\ ${ }^{3}$ Institute for Clinical and Experimental Medicine, Prague, Czech Republic
}

This abstract was presented as the Cellular and Molecular Nutrition Theme highlight.

Obesity is an excess of adipose tissue (AT) and is linked with increased inflammation that enhances risk of type-2 diabetes and cardiovascular disease ${ }^{(1,2,3)}$. The BIOCLAIMS Study assessed subcutaneous white AT (sWAT) inflammation in metabolically healthy obesity in which metabolic syndrome had not yet manifested, and responses to chronic supplementation with omega-3 fatty acid (FA) which are widely investigated for their anti-inflammatory actions ${ }^{(3)}$.

sWAT biopsies were collected pre- and post-12-week supplementation with $1.1 \mathrm{~g}$ EPA $+0.8 \mathrm{~g}$ DHA/day or corn oil. sWAT FA composition, lipid mediator profile, whole transcriptome expression, protein expression, morphology, and immune cell infiltration were assessed by gas chromatography, coupled UPLC-mass spectrometry, RNA-Sequencing, immunoblotting, and immuno-histochemical staining respectively.

Metabolically healthy obesity was associated with dysregulated FA and lipid mediator profiles exhibiting higher concentrations of arachidonic acid (AA) and respective oxylipins, lower concentrations of DHA oxylipins, and alteration of the endocannabinoid system (ECS) $(\mathrm{P}<0.05)$. Metabolically healthy obesity was further associated with altered transcriptome expression suggestive of enhanced inflammation, immune response, tissue remodelling, and expansion. This was concordant with tissue morphology in which metabolically healthy obesity was associated with adipocyte hypertrophy and presence of macrophages arranged in crown like structures $(\mathrm{P}<0.05)$; however, total and pericellular fibrosis was not altered in metabolically healthy obese individuals but was positively correlated with HOMA 2-IR $(\mathrm{P}<0.05)$. Chronic supplementation with EPA+DHA increased concentrations of sWAT omega-3 FAs and derived oxylipins $(\mathrm{P}<0.01)$, and decreased AA oxylipins, with particular effect on the ECS predominantly in normal weight individuals $(\mathrm{P}<0.05)$. EPA+DHA modulated the sWAT transcriptome suggesting promotion of tissue remodelling and down regulation of chronic inflammatory response $(\mathrm{P}<0.05)$.

These data suggest enhanced sWAT inflammation in the context of tissue expansion and remodelling is associated with metabolically healthy obesity. Furthermore, that sWAT of metabolically healthy obese individuals without type-2 diabetes maintains some degree of normal function in that it is still sensitive to dietary lipid manipulation. EPA+DHA are able to modulate synthesis of EPA, DHA and AA derived oxylipins and transcriptome expression but obesity may involve resistance to these effects particularly on the ECS.

\section{References}

1. Dewhurst-Trigg R, Hulston CJ \& Markey O (2020) Proc Nutr Soc 17, 1-15.

2. Boden G (2011) Curr Opin Endocrinol Diabetes Obes 18 (2), 139-143.

3. Macedo Rogero M \& Calder PC (2018) Nutrient 10 (4), 432. 